\title{
Del disciplinamiento de los cuerpos al gerenciamiento de la vida. Mutaciones biopolíticas en el presente en torno a la construcción de la anormalidad*
}

\author{
Martín E. Díaz**
}

\section{Resumen}

El siguiente artículo propone abordar las transformaciones que operan en el presente en relación con el gobierno de los cuerpos y la construcción de una otredad negada a partir de la mutación de un diagrama de poder disciplinario a una gubernamentalidad neoliberal.

En primer lugar, se pretende indagar, a partir de los aportes de Michel Foucault, el surgimiento de la biopolítica, en tanto estrategia de gobierno de las poblaciones tanto dentro como fuera de Europa, a efectos de analizar las estrategias discursivas que hicieron posible en el siglo XIX el surgimiento del par normal/patológico.

En segundo lugar, se propone examinar las transformaciones que se generan a partir del despliegue de la gubernamentalidad neoliberal y, con ello, la mutación del par normal/patológico como criterio de demarcación social. Así pues, la racionalidad neoliberal parece abrir paso a un gerenciamiento de la vida

\footnotetext{
* Artículo recibido el 25 de mayo de 2013. Aceptado el 09 de septiembre de 2013.

Quiero agradecer profundamente a Susana Murillo sus sugerencias y los intercambios que hicieron posible la escritura de este trabajo.

** Docente e investigador de la Facultad de Humanidades de la Universidad Nacional del Comahue (UNCo). Doctorando en Filosofía por la Universidad Nacional de Córdoba (UNC). Miembro del comité ejecutivo del Centro de Estudios y Actualización en Pensamiento Político, Decolonialidad e Interculturalidad (CEAPEDI), Neuquén, Argentina.
} 
planetaria sobre la base de la modelación de la conducta de los sujetos de acuerdo con las estrategias discursivas del mercado.

\title{
Palabras clave
}

Biopolítica - normal/patológico - gubernamentalidad neoliberal - gerenciamiento de la vida.

\begin{abstract}
The following article aims at boarding the transformations which take place nowadays in relation to body government, and the construction of an otherness useless to start from the mutation of a disciplinary power diagram into a neoliberal governmentality.

Initially, the idea is, based on Michel Foucault's contribution, to enquire the origin of biopolitics, as a governmental strategy for populations both inside and outside Europe, so as to analyse the discursive strategies that made possible in the $19^{\text {th }}$ Century the origination of the normal/pathological pair. On a second instance, this article aims at examining the transformations that generate as a result of the deployment of neoliberal governmentality and, with it, the mutation of this normal/pathological pair as a criterion of social delimitation. By these means, neoliberal rationality seems to leave place to planetary life managing on the basis of a sculpting attitude towards subjects' behavior in accordance with the market's discursive strategies.
\end{abstract}

\section{Keywords}

Biopolitics - normal/pathological - neoliberal governmentality - life managing.

\section{Introducción}

Vidas que son como si no hubiesen existido, vidas que sobreviven gracias a la colisión con el poder que no ha querido aniquilarlas o borrarlas de un plumazo.

M. Foucault

El punto de partida de este trabajo consiste en analizar el momento de emergencia ad intra de los Estados modernos europeos de lo que Michel Foucault ha denominado biopolítica y la relación que esta guarda con el surgimiento en el siglo XIX del par normal/patológico como criterio de demarcación social. 
En este sentido, se procura en primer lugar problematizar de qué modo el ingreso de la vida en la esfera del poder constituirá un acontecimiento crucial en la historia de Occidente, a partir del despliegue de una tecnología macrofísica que tendrá la potencialidad de delimitar entre aquello que debe vivir y aquello que debe morir en el interior de la población, en momentos en que los requerimientos del aparato productivo capitalista demandarán convertirla en una totalidad sana y vigorosa.

De tal modo, la emergencia del par normal/patológico en el siglo XIX puede ser leída como el resultado de este ejercicio del poder sobre la vida, que buscará defender a las poblaciones sanas y productivas de aquellos individuos que resulta menester resocializar o exterminar en nombre de la perdurabilidad de la especie.

En segundo lugar, interesa poner en tensión la mutación de este parámetro del par normal/patológico — preponderante durante el siglo XIX hasta mediados del siglo XXen nuestro presente, a partir de las nuevas tecnologías de gobierno y modos de existencia que se despliegan con la gubernamentalidad neoliberal.

Esta indagación reviste de importancia dado que se considera que las actuales tecnologías de poder, insertas en las nuevas dinámicas globales o geopolíticas del neoliberalismo, parecen abrir paso a novedosas formas de control y gerenciamiento de la vida planetaria sobre la base de la modelación y sanción de la conducta de los sujetos de acuerdo con las estrategias discursivas o mandatos del mercado.

\section{La emergencia de la biopolítica y su relación con la formación del par normal/patológico}

Frente a las dificultades y desafíos que habrán de presentarse en el interior del funcionamiento de las sociedades capitalistas modernas a partir del surgimiento en el siglo XVIII de la llamada 'cuestión social' ${ }^{1}$ (Donzelot, 2007) en Europa, la medicina pasará a ocupar un lugar privilegiado en la labor de intervenir en la vida de los individuos, y sobre todo de aquellos sectores sumergidos en la pobreza.

En este contexto histórico signado por un conjunto de transformaciones económicopolíticas producidas en el interior la sociedad liberal contractualista, la medicina moderna adquirirá una dimensión profundamente social al abocarse al cuidado de los cuerpos individuales y colectivos mediante la labor de diagnosticar y tratar los problemas que aquejan a este tipo de sociedades. Es así que la medicina moderna formada a partir de una matriz positivista - se convertirá en una estrategia discursiva

\footnotetext{
${ }^{1}$ Por 'cuestión social' debe entenderse el resultado del déficit producido entre aquello que acontece efectivamente en la vida concreta de los individuos y los imaginarios jurídico-políticos propuestos por un modelo contractualista que proclamaba la universalidad formal de los postulados de igualdad, libertad y derecho de propiedad. Lo 'social' emerge entonces como una estrategia de sujeción de los individuos a un ordenamiento socioeconómico —sostenido en la pauperización masiva de los sectores populares- en aras de evitar el estallido o desintegración del mismo (Donzelot, 2007).
} 
cuyo objetivo será 'curar' tanto las enfermedades físicas como morales que afectan a la población. En tal sentido, la enfermedad pasará a ser concebida como un 'problema social' que deberá ser correctamente diagnosticado y resuelto y, para ello, resultará menester conocer los procesos internos del cuerpo, organismo en que deviene la población mediante el conocimiento de sus ciclos de vida —reproducción, natalidad, morbilidad, etc.-, así como de los factores que afectan su salubridad - pestes, epidemias, enfermedades contagiosas, condiciones socioambientales, etc.- .

Este acontecimiento novedoso que representará la medicina moderna en el siglo XVIII se enmarcará en el desarrollo de una 'política de la salud' (Foucault, 1991) que tendrá como una de sus principales características producir un desplazamiento respecto de la labor de asistencia y auxilio dirigida a los pobres, tal como ocurría en los hospitales generales en la época clásica. La asistencia casi sacramental del 'pobre menesteroso' - a la cual se atribuía un carácter concluyente en el abordaje de la enfermedad - se verá reemplazada por una nueva mirada hacia la pobreza vinculada con los problemas de la ociosidad y con el desarrollo de los medios más eficaces para lograr que los individuos menesterosos se conviertan en una mano de obra útil dentro del aparato de producción económico propio de la sociedad liberal capitalista. Otras de las características principales que asumirá esta 'política de la salud' consistirá en la búsqueda por parte del poder político del 'bienestar' de la sociedad a través de la puesta en práctica de un conjunto de gestiones que permitirán intervenir sobre el cuerpo social a partir del conocimiento de las condiciones de vida, la cantidad de habitantes y actividades que desarrollan, la circulación de los productos generados por los hombres, etc. (ibídem).

El cuerpo individual y colectivo se convierte de esta manera en el foco de interés de la medicina moderna al convertir 'lo social' en su campo de intervención privilegiado. De aquí que la medicina moderna deviene en una medicina social cuya función será erigirse en una herramienta de prevención de las enfermedades atendiendo a los factores económicos y sociales que les dan origen.

Así pues, la promoción de la salud y el bienestar de la población irá acompañada de distintas estrategias de control social —como lo serán la corriente médico-política alienista, la filantropía y el higienismo-, que tendrán como propósito resguardar la salubridad poblacional de aquellos 'focos patológicos' que atentan contra el flujo de sujetos y mercancías que se requiere para el 'buen funcionamiento' de las cosas. En esta clave resulta posible hablar del despliegue de un proceso de medicalización de lo social $^{2}$ que permitirá al Estado moderno asumir como labor central tomar a su cargo la vida de las poblaciones.

\footnotetext{
${ }^{2}$ Foucault (1987) da cuenta de tres grandes momentos históricos de este proceso de medicalización de lo social vinculado con la conformación de los Estados modernos europeos. En primer término, el desarrollo de la 'medicina de Estado' en Alemania a comienzos del siglo XVIII; en segundo término, la formación de
} 
Ahora bien, ¿en qué consiste esta novedosa capacidad por parte del Estado moderno de tomar a su cargo la vida colectiva? Y, en relación con ello, ¿cómo se vincula este poder que se ejerce sobre la vida con la sanción del fenómeno de la anormalidad en el siglo XIX?

Dentro de las primeras indagaciones efectuadas por Foucault en torno al surgimiento de este poder sobre la vida, este aparecerá representado como una tecnología de dominación de las poblaciones que el filósofo francés analizará bajo la forma de una 'tecnología de doble faz' en el último capítulo de La Voluntad del saber (1987b) y como el despliegue de una tecnología superpuesta a los mecanismos de disciplinamiento y adiestramiento de los cuerpos en su curso del 75-76' ll faut défendré la societé, conocido con el nombre de Defender la sociedad (2000b).

Se trata para el autor en cuestión del despliegue de un proceso histórico que establecerá una novedosa función por parte del Estado moderno, caracterizada por convertir a la población en un objeto de cálculo y regulaciones, como asimismo de depuración biológica frente a aquellos elementos anómalos capaces de interferir en su óptimo funcionamiento.

De esta manera, el despliegue de este poder sobre la vida supondrá en la historia de Occidente una profunda transformación de la vida humana al convertirla en un objeto posible de ser manipulado, regulado y modificado de acuerdo con ciertas metas previamente prefijadas. Lo relevante de este análisis propuesto por el filósofo francés consiste en la visualización de la alianza indisociable que se establecerá entre este poder que se ejerce al nivel de la vida y el desarrollo del capitalismo de los siglos XVIII y XIX, al constituirse esta tecnología de poder en un elemento indispensable para el desarrollo del capitalismo y el ajuste de los cuerpos individuales y colectivos a los requerimientos de los procesos económicos puestos en marcha.

Como señalamos, el surgimiento de este poder ejercido sobre los cuerpos individuales y colectivos por parte del Estado moderno remitirá a dos tecnologías de poder imbricadas entre sí:

-una focalizada sobre los cuerpos individuales, que emergerá a lo largo de los siglos XVII y XVIII principalmente en Francia, con el desarrollo de las 'disciplinas' en el interior del ejército y la escuela (Foucault, 1985). Se trata de la generación de una serie de disciplinas que funcionará como una técnica de individuación al posibilitar la vigilancia de la conducta y los comportamiento de los individuos, intensificar su rendimiento, ubicarlo en los lugares donde pueda resultar más útil, etc. Este poder individualizante que Foucault (2000b) denomina 'anatomopolítica' se focalizará en los cuerpos de los individuos para hacer de ellos sujetos más dóciles y eficientes. 
- una tecnología de poder desarrollada a mediados del siglo XVIII en Inglaterra, que tendrá su foco de intervención no en una labor individualizante efectuada sobre los cuerpos, sino en un ejercicio totalizante desplegado sobre la población entendida como un corpus unitario. En este caso se trata de un poder focalizado en la totalidad de los procesos biológicos que hacen a la vida de la población con el propósito de convertirla en una 'máquina de producir' tanto riquezas como individuos. De esta manera, la salud, la higiene, como asimismo los flujos de crecimiento y descrecimiento que posee el corpus poblacional se convierten en objeto de una tecnología totalizante que Foucault (1987b, 2000b) designa con el nombre de 'biopolítica', la cual encontrará en la estadística un saber clave para la recolección de aquellos conocimientos necesarios que permitan convertir a la población en una totalidad sana y vigorosa.

De aquí que de acuerdo con el análisis que realizará Foucault en su clase del 17 de marzo del 76 en su mencionado curso Il faut défendré la societé, la emergencia a mediados del siglo XVIII de la biopolítica debe leerse como un proceso de mutación histórica que dejará atrás un poder de soberanía -inscripto en el modelo hobbesiano de un 'poder de espada' capaz de conceder la vida o establecer la muerte de sus súbditos- por un nuevo tipo de poder ejercido por el Estado moderno bajo el sintagma de un 'hacer vivir' o 'dejar morir' —'faire vivre' ou 'laissez mourir' - (Foucault, 2000b) a quienes gobierna. Este nuevo modelo de poder ejercido por el Estado moderno es lo que le permitirá optimizar la vida de ciertas franjas de la población y, en su reverso, administrar la muerte o producir el exterminio de aquellas franjas poblacionales consideradas peligrosas para el continuum biológico de la especie. De tal modo, esta potencialidad de 'hacer vivir' o 'dejar morir' -'faire vivre' ou 'laissez mourir' - a las poblaciones resultará posible para Foucault merced al surgimiento del racismo dentro de los Estados modernos, en tanto dispositivo de guerra que permitirá eliminar a las poblaciones indeseables tanto dentro como fuera de Europa.

En esta dirección el racismo moderno constituirá una estrategia de control y exterminio biopolítico aplicado tanto sobre las poblaciones indeseables ad intra de Europa como en la vida en las colonias, en el contexto de la consolidación imperial de las potencias hegemónicas europeas - tales como Inglaterra, Francia y Alemaniadurante los siglos XVIII y XIX. En este sentido, el despliegue del biopoder analizado por Foucault es posible de ser pensado como una tecnología de poder que operará en distintos niveles de complejidad, lo que permitirá actuar tanto a un nivel molecular, molar como global a partir del control de los recursos materiales y humanos disponibles del planeta (Castro-Gómez, 2007).

Sin pretender en esta oportunidad efectuar un análisis acerca de los alcances imperiales del biopoder (Díaz, 2012), así como de los aportes y posibles limitaciones de la analítica foucaultiana para comprender estos regímenes globales o tecnologías 
macrofísicas de gobierno de las poblaciones ${ }^{3}$, nos interesa puntualmente enfatizar de qué modo el racismo moderno configurará un dispositivo global que jugará un papel central en el exterminio del Otro o de la 'mala raza', como garantía de la perdurabilidad de aquellos individuos considerados deseables para la vida.

Así las cosas, el racismo moderno — vinculado aquí con los discursos del darwinismo y la eugenesia- poseerá un rol clave en la administración de la vida y la naturalización de la muerte mediante la apelación a una supuesta superioridad natural de ciertos hombres y naciones, fundada en aparentes razones científicas (Murillo, 2001). En otras palabras, biopolítica y tanatopolítica — política de la vida y política de la muerteconstituirán las 'dos caras' o el 'doble rostro' de una misma tecnología de gobierno que permitirá delimitar entre lo deseable para la vida y aquello que subvierte o amenaza la continuidad de ella (Mendieta, 2007).

De tal modo, la dimensión individualizante y totalizante que asumirá el Estado moderno respecto de los cuerpos individuales y colectivos tendrá como punto de intersección al sexo - o más bien la esfera de la sexualidad-, lo que permitirá al Estado moderno aglutinar las cuestiones relativas al cuidado de la salud pública, la progenie y el reguardo de la raza. En esta clave, el control de la sexualidad constituirá uno de los mecanismos privilegiados utilizado por la maquinaria estatal moderna en el propósito de establecer ciertos modos de existencia prototípicos, ajustados a las reglas de moralidad y las buenas costumbres instauradas. La sexualidad emerge entonces como el campo de batalla en donde van a librarse los modos en que los sujetos deberán constituirse en existencias hereditariamente sanas, socialmente útiles y potencialmente emprendedoras, o bien parafraseando a Félix Guattari (1996), en 'subjetividades capitalísticas' reproductoras de ciertos modos normalizados de ser sujetos.

Así pues, la sexualidad se convertirá en el blanco del poder mediante la generación a partir del siglo XVIII de un 'dispositivo de la sexualidad' ${ }^{4}$ que encontrará en el nuevo

\footnotetext{
3 De acuerdo con la lectura propuesta por Castro-Gómez (2007), Foucault entiende al colonialismo como un fenómeno intraeuropeo producido particularmente a fines del siglo XVIII y durante el siglo XIX. Algo similar ocurre con la idea de racismo moderno analizada por el filósofo francés, el cual no nace con la experiencia colonial europea sino que esta última es vista como un campo o laboratorio que posibilita el desarrollo del mismo. A diferencia de este planteo propuesto por Foucault, consideramos que el racismo resulta indisociable de la experiencia colonial europea puesta en marcha no en el siglo XVIII y XIX sino a partir de 1492 con el control geopolítico del Atlántico por parte de las entonces potencias hegemónicas europeas -España y Portugal- y la anexión de nuestro continente al sistema mundo moderno/colonial capitalista. En esta dirección, la vinculación entre colonialismo y racismo con lo que propende la analítica de Foucault parece conducir a un 'punto ciego', al obviar casi cinco siglos de experiencia colonial y el rol que cumplirá el racismo como estrategia de control de la vida en las colonias. Véanse al respecto, entre otros (Castro-Gómez, 2007; Quijano, 2001; Young, 1995).

${ }^{4}$ Este dispositivo de la sexualidad logró formularse para Foucault (1987) a partir de la pastoral cristiana y la penitencia de la confesión. En esta dirección, la ciencia sexual — scientia sexualis- desarrollada en el siglo XIX conservará el rito de la confesión generado por el cristianismo a partir del siglo XVI, el cual constituirá la primera técnica de producir la verdad acerca del sexo en Occidente.
} 
modelo de una familia medicalizadora un elemento táctico indispensable para su penetración, tanto al nivel molecular de los cuerpos como al nivel molar en que funciona la población. Por ello, la sexualidad deviene en el centro del problema económico-político de la población, en un asunto de 'ciencia de policía' o de política pública cuya finalidad consistirá en el mejoramiento y ordenamiento de las fuerzas colectivas e individuales (Foucault, 2006).

En este sentido, la emergencia de este dispositivo de la sexualidad convertirá como objetos privilegiados de su saber a la 'mujer histérica' —en razón de las obligaciones conyugales y maternales otorgadas a la mujer en tanto madre atenta y guardiana del hogar-, el 'niño masturbador' —a quien deberá resguardarse moral e intelectualmente dada su futura función de conservar su familia y la descendencia sana-, la 'pareja malthusiana' —relacionada con la responsabilidad que deberán asumir las parejas respecto del cuerpo social respecto de la fecundidad y el control de las nacimientosy, por último, el 'adulto perverso' —-sobre el que recaerá una psiquiatrización de su conducta en nombre de la protección de la raza y de la especie- (Foucault, 1987).

El desarrollo de este dispositivo de la sexualidad constituirá entonces una pieza central frente a los requerimientos de la maquinaria estatal moderna de regular los nacimientos y matrimonios, preservar la fecundidad de la especie, como asimismo combatir las enfermedades que puedan atentar contra la longevidad y salubridad de la población existente o bien de las futuras generaciones.

A los propósitos de preservar esta deseada salubridad y longevidad de la población es que los aspectos vinculados con el control de la herencia y las posibles degeneraciones que pueden transmitirse de generación en generación aparecerán como preocupaciones medulares, frente a las cuales vendrá a dar respuesta una medicina de las perversiones, sumada a los postulados de la selección reproductiva del programa eugenésico o 'ciencia del cultivo de la raza' esbozado en primer término en 1883 por Sir Francis Galton, primo de Charles Darwin (ibídem).

El punto axial entre esta medicina de las perversiones y el programa eugenésico se producirá por vía de la 'teoría de la degeneración' formulada en 1857 por Auguste Morel en Francia, la cual permitirá fundamentar la relación reversiva que existe entre el desarrollo de una herencia patológica y su incidencia en la generación de un perverso sexual, y el desarrollo de este tipo de perversión vinculado con el agotamiento de la descendencia y la esterilidad de la especie. De esta manera, el problema de la degeneración aparecerá representado como el efecto de la anomalía producida sobre el niño por los padres. De aquí que se calificará de degenerado a un niño sobre el cual pesan los restos de la locura de sus padres y ascendientes, dado que la anomalía conduce a la locura y la locura produce anomalía por la degeneración (Foucault, 2005).

En este contexto de despliegue de una tecnología individualizante y totalizante por parte del Estado moderno, que buscará a través del 'dispositivo de la sexualidad' intervenir sobre los cuerpos individuales y colectivos en pos de controlar su herencia y 
diagnosticar a tiempo los factores disgénicos que conducen a la enfermedad, es que emergerá un conjunto de mecanismos de control social que se propondrán auscultar los potenciales peligros que se esconden en el corpus poblacional y, con ello, detectar a las conductas 'desviadas' en relación con los parámetros de normalidad establecidos. Así las cosas, el tejido social quedará inmerso en un proceso de normalización y disciplinamiento de las conductas que permitirá demarcar entre aquel 'ciudadano normal' adaptado a las normas sociales y morales prefijadas y aquellos sujetos desviados de ellas.

En este sentido, el surgimiento en el siglo XIX del par normal/patológico debe entenderse, entonces, como el resultado de las intervenciones producidas sobre la población en aras de convertirla en un todo sano y productivo. Para el cumplimiento de dicho propósito será menester delimitar las conductas prefijadas dentro de los parámetros deseables para el buen funcionamiento de la sociedad y aquellas que al desviarse de dichos parámetros producen desajustes sociales o morales. Será función entonces de las instituciones disciplinarias modernas, tales como la familia, la cárcel, la escuela, el hospital, el manicomio, producir una resocialización de estas conductas con el objeto de volverlas más gobernables y adaptables a los requerimientos de una biopolítica liberal, y evitar con ello la generación de conflictos sociales que afecten su óptimo funcionamiento (Murillo, 2012).

A modo de síntesis de este apartado, la emergencia del biopoder constituirá el despliegue de una tecnología de gobierno de las poblaciones, la cual tendrá en el 'dispositivo de la sexualidad' y en el racismo como dispositivo de guerra las estrategias que posibilitarán resguardar y optimizar a ciertas vidas, así como matar, en nombre de la defensa de un 'nosotros', a una otredad considerada indeseable para la existencia colectiva. En este esquema del biopoder, la construcción de la anormalidad involucra siempre una desviación, una especie de peligro de muerte que acecha entre los vivos.

\section{La gubernamentalidad neoliberal y la mutación del par normal/patológico en el presente}

Los modos en los que se despliega y ejerce el biopoder en las sociedades contemporáneas, al igual que las formas en que el universo de la anormalidad es delimitado y sancionado, parecen ya no responder a los parámetros desarrollados en las sociedades disciplinarias y al par normal/patológico tal como era pensado en el interior de ellas.

Las investigaciones efectuadas por el filósofo francés en sus cursos del 77-78, Seguridad, territorio y población, y del 78-79, Nacimiento de la biopolítica, introducirán una nueva lectura acerca del funcionamiento de las racionalidades de gobierno desplegadas sobre las poblaciones a partir de la utilización del concepto de 
gubernamentalidad —gubernamentalité-, mediante el cual pasará a ser comprendido o analizado el campo de indagación de la biopolítica.

La analítica de la gubernamentalidad permitirá en esta clave indagar los modos en que las conductas, deseos y motivaciones de los sujetos son posibles de ser moldeados o modelados para el cumplimiento de ciertos fines o logros específicos, al igual que el desarrollo de una tecnología de poder basada en el 'gobierno de las acciones' de los sujetos, en contraste con el modelo bélico o 'hipótesis Nietzsche' utilizado por Foucault hasta su curso del 76 (Castro-Gómez, 2010). ${ }^{5}$

No es por cierto el propósito centrarnos en este trabajo en un análisis genealógico acerca de las condiciones históricas que posibilitaron la emergencia o 'desbloqueo' del neoliberalismo en tanto 'arte de gobierno' de las poblaciones. Más bien lo que nos interesa poner en tensión es de qué modo los acontecimientos históricos producidos en la sociedad occidental a mediados del siglo XX y las transformaciones socio-técnicas generadas a escala global por un capitalismo mundial integrado (Guattari, 2004) parecen dar cuenta del ingreso en una nueva fase del desarrollo del biopoder producido por la gubernamentalidad neoliberal, lo cual supondrá la emergencia de una refinada tecnología de autogobierno de los sujetos, así como un nuevo modo de delimitación de los potenciales 'peligros sociales' sancionados ahora desde la racionalidad del mercado.

Este capitalismo mundial integrado (CMI) genera al decir de Félix Guattari (2004) un nuevo reordenamiento global sobre la base de una serie de transformaciones de los modos de funcionamiento y acumulación de las anteriores formas de capitalismo de Estado, lo cual dará lugar a una red policéntrica compleja — carente de localización específica - capaz de desterritorializar las barreras nacionales a partir de los nuevos complejos informáticos, militar-industriales y los medios masivos de comunicación. Estas transformaciones globales producidas por el CMI traen como consecuencia la imposibilidad de diferenciar entre un 'adentro' y un 'afuera' -las nuevas dinámicas globales parecen no responder a la separación entre este/oeste, norte/sur-, lo cual no debe entenderse como una desaparición de las segmentaciones sociales que sostenían los modos de acumulación propios de la sociedad industrial capitalista. Más bien el

\footnotetext{
${ }^{5}$ El modelo de análisis desde el cual Foucault examinó la emergencia de la biopolítica en Occidente se basó hasta entrado el año 76 en el esquema de la lucha/represión o en un modelo bélico de las relaciones de poder que atraviesa a la totalidad del corpus poblacional. Desde este modelo analítico el surgimiento de la biopolítica será comprendido como el despliegue de una tecnología de dominación, lectura que se verá modificada en sus cursos del 78 y 79 al comprender a partir de entonces la biopolítica como una tecnología basada en el gobierno sobre las acciones de los sujetos. Como ha mostrado Castro-Gómez, Foucault abandonará la concepción bélica del poder en sus cursos Seguridad, territorio y población y Nacimiento de la biopolítica, en los cuales introducirá la noción de gubernamentalidad como campo empírico de posibilidad o grilla de inteligibilidad del ejercicio del biopoder. En esta dirección, utilizaremos en este segundo apartado el neologismo de gubernamentalidad neoliberal para hacer referencia a la racionalidad de gobierno propia del neoliberalismo y las formas de gobierno de las poblaciones y modos de existencia que se despliegan con él.
} 
despliegue del CMI produce para Guattari (2004) zonas de superdesarrollo y de superenriquecimiento en contraste con zonas signadas por una pauperización absoluta, lo que convierte a estas regiones del planeta en verdaderos campos de exterminio de la fuerza de trabajo. En este sentido, el CMI al que refiere Guattari da cuenta del desarrollo de las nuevas dinámicas de poder producidas a escala global y su la articulación en una red molar capaz de movilizar los deseos individuales o moleculares - a partir de modos cada vez más flexibles, veloces y eficientes - hacia la esfera del consumo.

En esta dirección, podría denominarse 'sociedades de control' a esta nueva fase del ejercicio de las tecnologías biopolíticas caracterizada por una 'acción a distancia' sobre los sujetos antes que un disciplinamiento directo sobre sus cuerpos y comportamientos (Lazzarato, 2006). Se trata de un tipo de sociedad definida por la proliferación de técnicas de seguridad, las cuales actúan sobre las virtuales acciones de los sujetos creando un entorno propicio, o bien un 'medio ambiente' —milieu - natural a través del cual se procura favorecer y regular cierto tipo de movilidad y conducta en los individuos.

Será entonces a partir de la década del 70 el momento histórico en que comenzará a producirse todo un conjunto de transformaciones que tendrán como foco garantizar el libre flujo de mercancías y el 'buen gobierno' de las poblaciones mediante el conocimiento de los comportamientos humanos y la creación de una especie de 'habitad natural' como lo será el mercado. ${ }^{6}$ En otras palabras, la década del 70 abrirá la puerta a escala global del despliegue del neoliberalismo, el cual no puede reducirse a una esfera estrictamente económica, sino que producirá un nuevo tipo de gubernamentalidad cuyo 'arte de gobierno' consistirá en dirigir las conductas de los sujetos de modo de establecer ciertos modos de existencia prefijados.

De tal forma, a partir de esta transformación a escala global antes mencionada, el modelo de sociedad actual ya no parece poseer como núcleo en el ejercicio de poder a las disciplinas - lo cual no implica que hayan desaparecido- ${ }^{7}$, y en relación con ello es posible visualizar una mutación del par normal/patológico como parámetro desde el cual entrever las nuevas modalidades de subjetivación en el presente.

\footnotetext{
${ }^{6}$ De acuerdo con Murillo (2012), si bien es posible identificar a la década del 70 como el momento de mutación histórica que hará posible el despliegue global del neoliberalismo, los inicios de este son posibles de rastrearse bastante tiempo atrás, teniendo como antecedente la década del 30, a partir de las reuniones efectuadas en Francia por el Ilamado 'Coloquio Lipmann' —aquí nacerá el término 'neoliberalismo-, en donde se pusieron en tensión las limitaciones que poseía el liberalismo para dar respuestas a los problemas relativos a la 'cuestión social'.

${ }^{7}$ Como ha advertido el propio Foucault (2006), no se trata de pensar el paso de una sociedad a la otra como el reemplazo de una 'era de lo disciplinario' por una 'era de la seguridad', sino más bien como el análisis de los mecanismos de poder puestos en juego en una sociedad y la otra en términos de ensamblajes maquínicos que asumen determinados modos predominantes en una época dada.
} 
Así las cosas, la racionalidad gubernamental neoliberal, generada a partir de las vertientes del ordoliberalismo alemán o el pensamiento económico de la Freiburger Schule y del neoliberalismo norteamericano de la Chicago School of Economics, producirán -con sus propias especificidades - en el derrotero del siglo XX (Foucault, 2007) modos de existencia sostenidos en la lógica del sujeto como 'empresario de sí mismo' o como 'capital humano', lo que permitirá estimular tanto el deseo de autogobierno como la competencia entre los individuos en el interior del 'régimen empresarial' en que deviene la llamada sociedad de control analizada por Gilles Deleuze (2006) en su Post-Scriptum.

En esta dirección, el autogobierno y autoinversión en sí mismo actúan como eficaces modelaciones de la subjetividad impulsada por el ethos empresarial que conlleva la gubernamentalidad neoliberal al promover el entrenamiento, la inversión en educación, el cuidado de la salud, la 'vigilancia molecular' (Rose, 2001) en pos de que los sujetos internalicen la necesidad de maximizar los beneficios y de minimizar los riesgos que conllevan sus acciones y decisiones.

De tal modo, el autocuidado y la autoinversión resultan las claves a partir de las cuales se establece el éxito y el fracaso de individuos inmersos en una competencia constante en aras de garantizar la propia autorrealización en un mundo que aparece representado como un juego de competencias ilimitado, en el que los sujetos intervienen de acuerdo con sus propias capacidades y deseos.

En virtud de esta incentivación al autocuidado y autoinversión de sí mismo fagocitados por la lógica del mercado, su reverso resultarán aquellos sujetos que desde el prisma de la racionalidad neoliberal- poseen una autoadministración deficiente de su propio capital o capacidades, lo cual explica las razones de su fracaso.

En este sentido, nos interesa remarcar una de las estrategias de gobierno de las poblaciones desplegadas por la racionalidad neoliberal, puesta en marcha por organismos internacionales como la Organización Mundial de Comercio, el Fondo Monetario Internacional y fundamentalmente el Banco Mundial, tendiente a delimitar los nuevos potenciales 'peligros sociales' a partir de la introducción de la idea de 'grupos de riesgo', los cuales es menester gestionar.

La idea de 'grupo de riesgo' no supone estrictamente la existencia de individuos anormales a quienes re-socializar o 'reencauzar', producto de su deviación a las normas establecidas - tal como ocurría en el interior de las sociedades disciplinarias-, sino de grupos que representan desde la perspectiva del mercado una mayor o menor potencialidad de alterar la gobernabilidad y el buen funcionamiento de aquel (Murillo, 2006). Así pues, estos grupos son monitoreados y controlados a fin de diseñar estrategias de prevención o extinción, según el potencial de riesgo que representan. De esta manera, la propuesta de 'gestionar el riesgo' involucra promover, en el orden de lo deseable, el permanente deseo en los sujetos de modos de existencia caracterizados 
por la flexibilidad, la movilidad, el autocuidado de la salud y la responsabilidad por la prevención de riesgos a futuro.

Tal como ha mostrado Murillo (2006) en su investigación documental en torno a los informes del Banco Mundial (BM) y la aplicación de las estrategias diseñadas para América Latina y en particular para Argentina, la gestión del llamado 'riesgo social' aparece fuertemente vinculada para el BM con el control de la pobreza o bien con los riesgos que acarrean los pobres para el funcionamiento del orden internacional.

En esta clave, la 'gestión de la pobreza' aparece como uno de los mayores objetivos de las estrategias promovidas por el BM en relación con el 'buen funcionamiento' del mercado, dado que los pobres son visualizados como 'grupos vulnerables' que poseen pocas o escasas oportunidades de autogestionarse a sí mismos y de contribuir mediante la competencia individual a la riqueza colectiva. Por ello estos grupos vulnerables - a los cuales se suman otros grupos de riesgo, como los toxicómanos y los 'adolescentes violentos' - conllevan un potencial riesgo en virtud de conformar una franja poblacional -o una underclass como lo denominó Gary S. Becker- con tendencias a los embarazos precoces y a la dependencia del Estado.

De tal modo, la pobreza aparece representada desde las estrategias de la racionalidad de mercado como producto de la 'incapacidad' por parte de ciertos sujetos de autogobernarse a sí mismos. En otras palabras, la pobreza emerge como el resultado de la 'mala previsión' o la 'ineficiente autoadministración' por parte de algunos sujetos, y no así como consecuencia de un orden global estructurado sobre una exclusión y marginación social colosal de amplísimos sectores de la población mundial.

Así las cosas, el gobierno de la pobreza parece asumir —en el caso particular de América Latina - ciertas modalidades específicas por parte de la racionalidad de mercado o gubernamentalidad neoliberal, caracterizadas por gerenciar la vida de aquellas franjas de la población más empobrecidas a partir de la promoción del 'desarrollo humano' de estos amplios sectores populares de 'Nuestra América' a 'niveles mínimos biológicos básicos', lo que ubica a estos sectores en las fronteras o umbrales de la muerte (Alvarez Leguizamón, 2008).

Siguiendo en esto a Sonia Alvarez Leguizamón (2008), puede denominarse 'focopolítica' al modo particular en que la gubernamentalidad neoliberal impactará sobre las políticas sociales de los países de América Latina. El concepto de 'focopolítica' remite a nuevas formas de neofilantropía y de caridad laica o religiosa que actúan sobre aquellos grupos sociales que se encuentran en situaciones de vulnerabilidad o de precarización de las condiciones existenciales elementales, lo que conduce a una acción tutelar sobre ellos, así como a la identificación de estas poblaciones de riesgo excluidas del mundo del trabajo. De esta manera, se procura fomentar por parte de estos grupos vulnerables formas de autocontrol mediante la promoción - principalmente por parte de instituciones gubernamentales que deben 'dejar hacer' según la naturaleza del 
mercado- de redes productivas de autogestión en pos de permitir una subsistencia básica y evitar situaciones de conflictos que alteren el orden social global establecido.

La construcción de un imaginario social que asocia a la pobreza con el fracaso individual y con la peligrosidad que representan estas underclass para la seguridad de los ciudadanos consumidores conduce a una estigmatización o 'criminalización de la miseria' a través de un perverso mecanismo que impide identificar las causas estructurales de la pauperización de vastos sectores de la población mundial y que conduce además a un profundo sufrimiento psíquico y social de estas vidas estigmatizadas.

\section{Algunas palabras finales}

Hemos propuesto en este trabajo indagar la construcción del fenómeno de la anormalidad pensándolo no como una realidad dada o un objeto acabado que asume naturalmente el nombre o la forma de lo 'anormal', sino más bien como el resultado de relaciones de poder específicas en las cuales ciertos cuerpos resultan negados en razón de la peligrosidad que se considera conllevan para la vida colectiva.

De tal modo, entendemos que la formación del par normal/patológico en el interior de la sociedad disciplinaria debe leerse como la necesidad de generar cuerpos dóciles y productivos que asuman como propios los requerimientos de una sociedad liberal capitalista, que tenderá a naturalizar las desigualdades sociales que se producen como condición para su buen funcionamiento. La construcción del anormal decimonónico responde entonces a ese sujeto que hay que resocializar para adaptarlo a las normas sociales y morales de una sociedad que procura defenderse de aquellas conductas capaces de atentar contra el orden instituido.

La mutación de este parámetro disciplinario de lo normal/patológico que consideramos se produce en el presente va de la mano con el despliegue de la gubernamentalidad neoliberal $y$, con ello, la generación de una nueva delimitación del tejido social que ya no parece responder a la tensión entre un 'ciudadano normal' y un 'anormal indisciplinado', sino a la contraposición entre un 'sujeto-exitosoautoadministrador' de su propia vida y un 'pobre-incapaz-peligroso' para la vida del resto.

De esta manera, la gubernamentalidad neoliberal, mediante la constante incentivación al éxito personal y a la 'autoinversión en sí mismo', parece abrir las puertas a los peligros de un neomalthusionismo y de un nuevo darwinismo social basado en la constante competencia por los recursos y los logros disponibles en el universo del mercado.

Así pues, la modelación de una subjetividad centrada en una competencia incesante por los logros disponibles en el interior del 'régimen empresarial' en que devienen nuestras sociedades contemporáneas conduce no solo a la imposibilidad de establecer 
lazos sociales que posibiliten una transformación del mundo social establecido, sino también a la naturalización del sufrimiento y la muerte de quienes son vistos como responsables de su propio fracaso.

\section{BIBLIOGRAFÍA}

Castro-Gómez, S. (2007). Michel Foucault y la colonialidad del poder, En Revista Tabula Rasa. N. ${ }^{\circ}$ 6, pp. 1-23.

(2010). Historia de la gubernamentalidad. Razón de estado, liberalismo y neoliberalismo en Michel Foucault. Bogotá: Coedición Siglo del Hombre editores, Pontificia Universidad Javeriana - Instituto Pensar y Universidad Santo Tomas.

Deleuze, G. (2006). Post-Scriptum sobre las sociedades de control. En Conversaciones. Valencia: Pre-Textos.

Díaz, M. (2012). Saberes científicos, racismo y eurocentrismo. Dispositivos imperiales en el gobierno de las poblaciones. En Díaz, M. y Pescader, C. (Comps.), Descolonizar el presente. Ensayos críticos desde el sur (pp. 55-76). Gral. Roca: Publifadecs, Universidad Nacional del Comahue (UNCo).

Foucault, M. (1985). Vigilar y castigar. El nacimiento de la prisión. México: Siglo XXI. (1986). Historia de la sexualidad. Tomo II El uso de los placeres. España: Siglo XXI. Ensayos.

(1987a.). La vida de los hombres infames. Buenos Aires: Caronte (1987b). Historia de la sexualidad. Tomo I La voluntad de Saber. México: Siglo XXI. Madrid: La Piqueta. (1991). La política de la salud en el Siglo XVIII. En Saber y Verdad. (2000a.). Los anormales. Curso en el Collège de France (1974-1975). Buenos Aires: FCE. (2000b). Defender la sociedad. Buenos Aires: FCE. (2005). El poder psiquiátrico. Curso en el Collège de France (1973-1974).

Buenos Aires: FCE. (2006). Seguridad, territorio y población. Curso en el Collège de France (1977-1978). Buenos Aires: FCE. (2007) Nacimiento de la biopolítica. Curso en el Collège de France (19781979). Buenos Aires: FCE.

Quijano, A. (2001). Colonialidad del poder, cultura y conocimiento en América Latina. En Mignolo, W. (Comp.), Capitalismo y geopolítica del conocimiento. El eurocentrismo y la filosofía de la liberación en el debate intelectual contemporáneo (pp. 117-131). Buenos Aires: Ediciones del Signo. 
Guattari, F. (1996). Caosmosis. Madrid: Traficantes de sueños. (2004) Plan sobre el planeta. Capitalismo mundial integrado y revoluciones moleculares. Madrid: Traficantes de sueños.

Lazzarato, M. (2006). Políticas del acontecimiento. Buenos Aires: Editorial Tinta Limón. Leguizamón Alvarez, S. (2008). Focopolítica y Gubernamentalidad Neoliberal, las políticas sociales. II Encuentro Argentino y Latinoamericano Prácticas sociales y Pensamiento crítico. Escuela de Trabajo Social, Universidad Nacional de Córdoba, 4-5 de abril, (paper).

Mendieta, E. (2007). 'Hacer vivir y dejar morir’: Foucault y la genealogía del racismo. En Revista Tabula rasa. N. ${ }^{\circ}$ 6, pp. 138-152.

Murillo, S. (Coord.). (2006). Banco Mundial. Estado, mercado y sujetos en las nuevas estrategias frente a la cuestión social. Buenos Aires: Ediciones del Centro Cultural de la Cooperación Floreal Gorini.

(2001). La ciencia aplicada a políticas sanitarias en Argentina y su relación con la escuela de Medicina de la Universidad de Buenos Aires (1869-1905). Buenos Aires, mimeografía.

(2012). Prácticas científicas y procesos sociales. Una genealogía de las relaciones entre ciencias naturales, ciencias sociales y tecnologías. Buenos Aires: Biblos.

Rose, N. (2001). Normality and Pathology in a biological age. En Outlines, N. ${ }^{\circ} 1$, pp. 19-33.

Young, R. (1995). Foucault on race and colonialism. En New Formations 25, pp. 1-18. 